\title{
Collisional activation of asteroids in cometary orbits ${ }^{\star}$
}

\author{
C. G. Díaz ${ }^{2}$ and R. Gil-Hutton ${ }^{1,2}$ \\ ${ }^{1}$ Complejo Astronómico El Leoncito - CONICET - Av. España 1512 sur, J5402DSP San Juan, Argentina \\ e-mail: rgilhutton@casleo.gov.ar \\ 2 Universidad Nacional de San Juan - Av. J. I. de la Roza 590 oeste, 5400 Rivadavia, San Juan, Argentina \\ e-mail: siempregonza@hotmail.com
}

Received 12 December 2007 / Accepted 27 April 2008

\section{ABSTRACT}

\begin{abstract}
Aims. We study the time an asteroid in a cometary orbit must wait to receive a collision producing a crater depth enough to expose subsurface volatiles, aiming to analyze the possibility of collisional reactivation of these objects if they are dormant comets. Methods. We perform a numerical integration of the asteroids in cometary orbits and a population of projectiles to find the mean intrinsic collision probabilities and mean impact velocities of the targets. The projectile population was obtained as a sample with the same distribution of orbital elements as observed for main belt asteroids, and we also take into account that its size distribution changes for different size ranges. Only 206 asteroids in cometary orbits, that are not members of other asteroid groups, with a Tisserand parameter $2 \leq T_{\mathrm{J}} \leq 2.9$ and perihelion distance $q>1.3$ AU were considered.

Results. A large fraction of the objects in the sample receive at least 1 collision energetic enough to break the comet crust and allow a dormant comet to reach an active state in a period shorter than a Jupiter Family Comet dynamical lifetime. A large fraction of the objects in the sample with $r_{\mathrm{t}} \geq 8-9 \mathrm{~km}$ receive several collisions and could be active for more than $3 \times 10^{4} \mathrm{yr}$. We found an excess in the number of dormant comet candidates from the expected values which is indicative of the presence in the ACOs population of objects that are not comets in a dormant state. These objects could be asteroids with $T_{\mathrm{J}}<3$ that reach their present orbits by some dynamical mechanism that perturbs the original asteroidal orbit changing its Tisserand invariant.
\end{abstract}

Key words. minor planets, asteroids - comets: general - solar system: general

\section{Introduction}

The different formation regions of asteroids and comets in the Solar System produced differences in their physical properties. Asteroids are planetesimals formed during the early ages of the Solar System in the region between Mars and Jupiter while comets were formed in a region that extends from the giant planets to the outer limits of the pre-solar nebula. As a consequence, there is a significant difference in the content of volatile material in both populations. This fact has provided the most apparent distinction between members of these two populations: comet nuclei, when close to the Sun, are usually surrounded by a coma produced by the out-gassing of volatiles caused by solar heating, while asteroids are not. This simple distinction has some complications due to the discovery of icy objects that rarely develop a coma due to their distance from the Sun, the discovery of asteroids with dynamical properties similar to those of comets, and the discovery of objects in typical asteroidal orbits that show temporary comet-like activity.

At the end of their active life some comets might develop an asteroidal appearance when sublimation stops and reaches dormancy or extinction due to the depletion of volatile material or by a crust built up on their surfaces (Rickman et al. 1990; Kührt \& Keller 1994; Benkhoff \& Huebner 1996; Jewitt 2002). The gas activity of a cometary nucleus coming close to the Sun can form a layer of dust grains that are too heavy to be blown off by the gas outflow. This crust could eventually become so thick that subsurface volatiles cannot be warmed up to sublimation

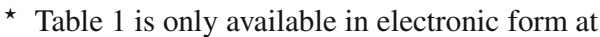
http: //www . aanda. org temperature, ceasing any cometary activity, and the nucleus appears observationally identical to an asteroid. Since this crust does not completely stop the vapor production in the comet interior, it could be possible that a large amount of ice is present below it even though the comet reaches an inactive state (Prialnik \& Mekler 1991).

One way to reactivate dormant comets is by means of impacts with interplanetary bodies. Fernández (1990) and Matese \& Whitman (1994) tried to explain the outburst activity of comets by impacts with small asteroids or meteoroids, and some authors have the opinion that this is the case for comets 41P/Tuttle-Giacobini-Kresák (Kresák 1974; Fernández 1981), 72P/Denning-Fujikawara (Beech 2001), and 133P/Elst-Pizarro (Toth 2000), while Toth (2001) suggested that the splitting of the comet C/1994 S4 (LINEAR) was a result of a collision of the comet with asteroid debris. Collisions of interplanetary boulders with a dormant comet could result in craters that partially destroy the crust on its surface, triggering reactivation by allowing the fresh material buried below the crust to begin sublimation in the next perihelion passage.

The dynamical criterion used to define the sample of objects that are candidate dormant comets is related to the Tisserand parameter (Kresák 1979), which is defined by the relation $T_{\mathrm{J}}=$ $a_{\mathrm{J}} / a+2 \cos I \sqrt{\left(a / a_{\mathrm{J}}\right)\left(1-e^{2}\right)}$, where $a$ and $a_{\mathrm{J}}$ are the semimajor axis of the orbits of the object and Jupiter, respectively, while $e$ and $I$ are the eccentricity and inclination relative to the orbital plane of Jupiter of the object's orbit. By this criterion, cometary orbits are defined as those having $T_{\mathrm{J}}<3$, while asteroidal orbits are those with $T_{\mathrm{J}}>3$. Therefore, all the objects with $T_{\mathrm{J}}<3$ that do not present any signature of cometary activity are defined as 
an asteroid in a cometary orbit (ACO). Objects with $T_{\mathrm{J}}<2$ have been called Damocloids by Jewitt (2005) and are asteroids in Halley-type cometary orbits, while those with $2 \leq T_{\mathrm{J}} \leq 3$ have orbits similar to the Jupiter family comets (JFCs). Therefore, ACOs are good candidates to be extinct or dormant comets.

Licandro et al. (2005) found differences in the spectroscopic properties of two sub-samples of ACOs, the near Earth objects (NEOs) with perihelion distance $q \leq 1.3 \mathrm{AU}$ and the non-NEOs, these last objects being spectroscopically similar to cometary nuclei. These authors also found that ACOs with featured spectra typical of the main belt have $T_{\mathrm{J}} \geq 2.9$ while those with $T_{\mathrm{J}}<2.9$ shown comet-like spectra, suggesting that the subsample of ACOs with $2.9 \leq T_{\mathrm{J}} \leq 3.0$ could be contaminated by a large fraction of interlopers from the inner part of the belt. On the other hand, Alvarez-Candal \& Licandro (2006) found that the sub-sample of ACOs with $q>1.3$ AU has a size distribution similar to that of the Jupiter family comets and can be composed of a significant fraction of dormant comets, while a large fraction of ACOs with $q<1.3$ AU could be scattered objects from the outer main belt.

The purpose of this paper is to analyze the possibility of reactivation of dormant comets by collisions with interplanetary boulders. Since the physical properties of JFCs are better known than for other comets and taking into account the above considerations, we only consider as dormant comet candidates ACOs with $2 \leq T_{\mathrm{J}} \leq 2.9$ and $q>1.3$ AU. In Sect. 2 we describe the computational method used and in Sects. 3 and 4 we present and discuss our results. In Sect. 5 we present our conclusions.

\section{Computational method}

In order to break the cometary crust and allow the dormant comet to reactivate in the next perihelion passage, it is necessary to break the target surface with impact craters deep enough to reach the buried ice. The crater diameter $D_{\mathrm{c}}$ produced by a collision with a projectile of radius $r_{\mathrm{p}}$ and impact velocity $v_{\mathrm{p}}$ is found using the expression proposed by Zahnle et al. (1998):

$D_{\mathrm{c}}=1.70 r_{\mathrm{p}}^{0.78} g^{-0.22} v_{\mathrm{p}}^{0.44}\left(\frac{\rho_{\mathrm{p}}}{\rho_{\mathrm{t}}}\right)^{0.333}(\cos \theta)^{0.44}$,

which is essentially the expression recommended by Schmidt \& Housen (1987) but considering that only the normal component of the impact velocity contributes to cratering. In this equation $\rho_{\mathrm{t}}$ and $\rho_{\mathrm{p}}$ are the densities for the comet and projectile, respectively, $g$ is the surface gravity on the comet, $\theta$ is the incidence angle measured from the zenith, and the equation must be evaluated in cgs units. The mean value of $\theta$ for the normal component of isotropic velocities is $45^{\circ}$ and we always assume densities of $\rho_{\mathrm{t}}=0.5 \mathrm{~g} \mathrm{~cm}^{-3}$ and $\rho_{\mathrm{p}}=2.5 \mathrm{~g} \mathrm{~cm}^{-3}$ for ACOs and projectiles, respectively. The exposed area produced by the crater is:

$A_{\mathrm{c}}=\pi\left(\frac{D_{\mathrm{c}}^{2}}{4}+h_{\mathrm{c}}^{2}\right)$

where $h_{\mathrm{c}}$ is the crater depth. If the crust thickness is $h$, to reach the ice below the crust we need $h_{\mathrm{c}} \geq h$ and using the crater depth/diameter ratio, which is almost constant for simple craters and equal to $0.18-0.20$ for the Moon and icy Galilean satellites (Schenk et al. 2004), it is possible to find the radius $r_{\mathrm{p}}$ of the projectile needed to form such crater.

On the other hand, the mean number of impacts received by the comet with projectiles of radius larger than $r_{\mathrm{p}}$ in a time $\Delta t$ is:

$$
\begin{aligned}
\left\langle N_{\text {col }}\left(>r_{\mathrm{p}}\right)\right\rangle & =\left\langle P_{\mathrm{i}}\right\rangle\left(r_{\mathrm{t}}+r_{\mathrm{p}}\right)^{2} \Delta t N_{\mathrm{pro}}\left(>r_{\mathrm{p}}\right) \\
& \approx\left\langle P_{\mathrm{i}}\right\rangle r_{\mathrm{t}}^{2} \Delta t N_{\mathrm{pro}}\left(>r_{\mathrm{p}}\right),
\end{aligned}
$$

where $r_{\mathrm{t}}$ is the comet radius, $\left\langle P_{\mathrm{i}}\right\rangle$ is the mean intrinsic collision probability between the comet and the projectile population, $N_{\text {pro }}\left(>r_{\mathrm{p}}\right)$ is the number of projectiles in the population with radius larger than $r_{\mathrm{p}}$, and $r_{\mathrm{p}} \ll r_{\mathrm{t}}$. The comet radius has been computed from the absolute magnitude of the object, $H$, by:

$\log \left(p_{v} \pi r_{\mathrm{t}}^{2}\right)=16.85+0.4\left(m_{\odot}-H\right)$

where the result is in kilometers, $m_{\odot}=-26.77$ is the apparent visual magnitude of the Sun, and a standard albedo $p_{v}=0.04$ was assumed.

Mean intrinsic collision probabilities and mean impact velocities can be inferred from statistical studies of the occurrence of orbital encounters between the comet and the projectile population, so we decided to estimate these parameters using the numerical method developed by Marzari et al. (1996). In this method the target and a projectile population were numerically integrated over a time span $T_{\text {int }}$ and the encounter distance and encounter velocity between the target and any projectile were recorded. Since Marzari et al. showed that the distribution of the cumulative number of encounters for an encounter distance less than $d_{\mathrm{enc}}$ is proportional to $d_{\mathrm{enc}}^{2}$, a distribution of the form:

$N_{\mathrm{enc}}\left(<d_{\mathrm{enc}}\right)=P_{1} \times d_{\mathrm{enc}}^{2}$

was assumed. $P_{1}$ is found by a fit to the data, taken as the standard deviation for each point $\sqrt{N_{\text {enc }}}$. Then, the mean intrinsic collision probability is obtained from:

$$
\left\langle P_{\mathrm{i}}\right\rangle=\frac{P_{1}}{n_{\text {pair }} T_{\text {int }}},
$$

where $n_{\text {pair }}$ is the number of different pairs of objects that can be formed within the interacting population.

Since the asteroid belt is the main source of projectiles, it is enough to use as the interacting population a sample of particles with the same orbital element distribution as that observed for the objects in the asteroid belt. This sample was obtained as follows: first, the objects that form the complete known asteroid population, i.e., those asteroids with mean apparent opposition $V$ magnitudes $V(a, 0)<15.75$ (Tedesco et al. 2005), and with semimajor axis $a>2 \mathrm{AU}$ were taken from the ASTORB database (ftp://ftp. lowell .edu/pub/elgb/ astorb . html) to get a first sample of 4549 objects. Then, a sixdimensional distribution of the orbital elements was calculated and a final sample of 350 particles was obtained at random from it. The hybrid integrator EVORB (Fernández et al. 2002) was used for the numerical integration of the targets and the particles of the interacting population, under the gravitational influence of the Sun and the planets from Mercury to Neptune. The integration was performed over a time span of $T_{\text {int }}=10^{5} \mathrm{yr}$, and a encounter was recorded every time the mutual distance between the target and a particle was less than $0.05 \mathrm{AU}$.

To find a value for $N_{\text {pro }}\left(>r_{\mathrm{p}}\right)$ it is necessary to know the cumulative size distribution of the real projectile population, i.e. the size distribution of the main asteroid belt. We assume an exponential size distribution of the form $\mathrm{d} N_{\text {pro }}(>r) \propto r^{-b} \mathrm{~d} r$, where $b$ is a characteristic exponent. Taking into account that the main belt size distribution changes for different size ranges, we have:

$$
\begin{aligned}
N_{\text {pro }}\left(>r_{\mathrm{p}}\right)= & N_{\text {pro }}(>500 \mathrm{~m}) \\
& +K_{0} \int_{r_{\mathrm{c}}}^{500 \mathrm{~m}} r^{-b_{0}} \mathrm{~d} r+K_{1} \int_{r_{\mathrm{p}}}^{r_{\mathrm{c}}} r^{-b_{1}} \mathrm{~d} r
\end{aligned}
$$


where $N_{\text {pro }}(>500 \mathrm{~m})=1.36 \times 10^{6}$ is the number of objects in the asteroid belt with radius larger than $500 \mathrm{~m}$ (Farinella \& Davis 1992; Tedesco \& Desert 2002; Morbidelli \& Vokrouhlický 2003; Bottke et al. 2005). The size distribution of very small main belt asteroids is not well known, so it is not easy to choose values for the parameters $r_{\mathrm{c}}, K_{0}, b_{0}, K_{1}$, and $b_{1}$. We decide to use two size distributions with $r_{\mathrm{c}}=200 \mathrm{~m}$ and $100 \mathrm{~m}$, respectively, and to assume the size distribution proposed by Yoshida \& Nakamura (2007) in the size range $r_{\mathrm{c}}<r<500 \mathrm{~m}\left(b_{0}=2.29\right)$, and a Dohnanyi (1969) size distribution for objects smaller than $r_{\mathrm{c}}$ $\left(b_{1}=3.5\right)$. Using this combined size distribution the total number of objects with a radius larger than $0.5 \mathrm{~m}$ is $1.42 \times 10^{13}$ in the first case and $6.11 \times 10^{12}$ in the second.

\section{Results}

The minimum ice exposed area needed to consider the comet as active could be obtained from the known JFC population. Almost all the studied JFCs have a fraction of active surface area below $\sim 10-20 \%$, with a large fraction of active area for the smaller comets and a very small one for the few observed comets with $r_{\mathrm{t}}>3 \mathrm{~km}$ (Tancredi et al. 2006). This could be indicative of a condition of the minimum effective exposed area to consider a comet as active, which could be proportional to $r_{\mathrm{t}}^{-2}$. Thus, we assume that a comet could reach an active state during the next perihelion passage when its exposed area is at least $10 \%$ of the total surface area of an object with radius $1 \mathrm{~km}$ $\left(1.257 \mathrm{~km}^{2}\right)$. With this definition an object must have a radius larger than $0.316 \mathrm{~km}$ to have the possibility of being active, because for smaller objects its total surface area is always less than the required exposed one.

There are different values for the mantle thickness in the literature, ranging from a few millimeters to several centimeters. Since we must be sure that the crater excavates the surface enough to reach the ice below it, only craters with a minimum depth of $h=1 \mathrm{~m}$ are considered to calculate the exposed area.

A sample of 206 dormant comets candidates was obtained from the ASTORB database, considering only ACOs with $2 \leq$ $T_{\mathrm{J}} \leq 2.9$ and $q>1.3 \mathrm{AU}$, and excluding objects with $3.03 \mathrm{AU}<$ $a<3.70 \mathrm{AU}, e<0.4$ and $i<25^{\circ}$ (possibly main belt or Cybele asteroids), with 3.70 AU $<a<4.20 \mathrm{AU}, e<0.4$ and $i<20^{\circ}$ (Hildas), and with $5.00 \mathrm{AU}<a<5.40 \mathrm{AU}$, and $e<0.3$ (Trojans). The absolute magnitude and radius of the ACO, mean intrinsic collisional probability, mean collision velocity and its error, the radius of the projectile that produces a crater with the minimum exposed area, and the times needed to receive such a collision for the two projectile size distributions $\left(r_{\mathrm{c}}=200 \mathrm{~m}\right.$ and $100 \mathrm{~m}$, respectively) are listed in Table 1 . The errors of the mean intrinsic collision probabilities are always less than $10^{-22} \mathrm{~km}^{-2} \mathrm{yr}^{-1}$ for all the objects. Twenty seven ACOs (5164, 30512, 32511, 37117, 96177, 1983JZ $, 1995 \mathrm{WL}_{3}, 2000 \mathrm{AU}_{242}$, $2000 \mathrm{QD}_{181}, 2000 W T_{168}, 2000 X O_{8}, 2001 Q G_{288}, 2003 B M_{1}$, $2003 U R_{267}, 2004 B T_{1}, 2004 K Z_{7}, 2004 R W_{141}, 2005 E B_{127}$, $2005 J_{173}, 2005 N X_{43}, 2005 X K_{57}, 2005 ; Y W_{24}, 2006 B V_{7}$, $2006 \mathrm{HS}, 2006 \mathrm{HP}_{131}, 2006 \mathrm{JO}_{65}$, and $2006 \mathrm{~S} \mathrm{O}_{134}$ ) had one or more close encounters with the planets and escaped before the integration ended. Then, their mean intrinsic collisional probability and mean collision velocity were calculated over a shorter time span, as indicated in Table 1.

\section{Discussion}

If the ACOs in our sample are dormant comets from the Jupiter family, their dynamics are also dominated by close encounters

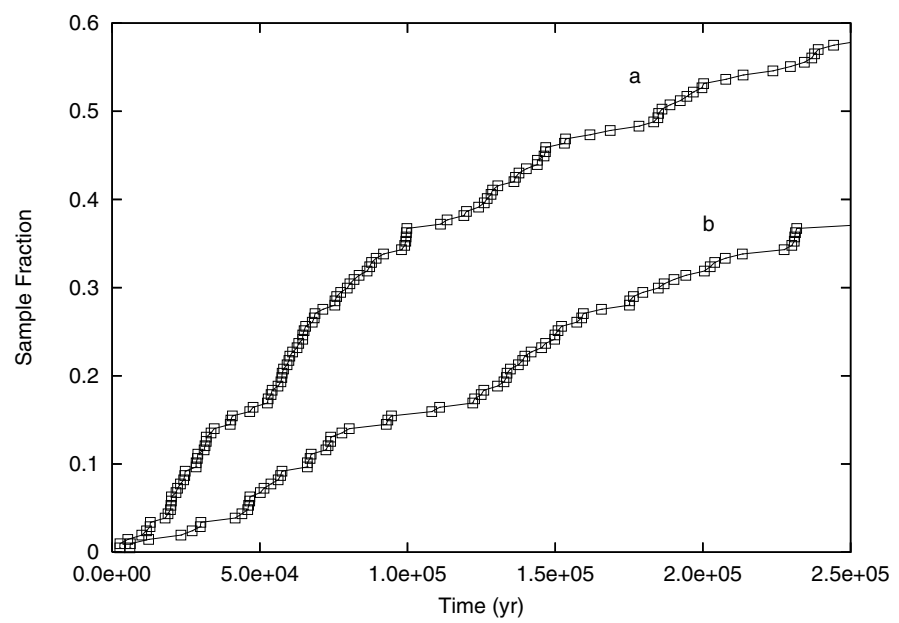

Fig. 1. Fraction of ACO sample reaching the minimum exposed area to be considered as active comets in a certain time, for a projectile size distribution with a) $r_{\mathrm{c}}=200 \mathrm{~m}$, and b) $r_{\mathrm{c}}=100 \mathrm{~m}$. Only times shorter than the dynamical lifetime of Jupiter family comets are considered.

with this planet producing strong perturbations in their orbits. Fernández et al. (2002) found that the dynamical lifetime of Jupiter family comets is $\sim 2 \times 10^{5} \mathrm{yr}$, which are relatively short compared to other populations. Assuming that this dynamical lifetime is also valid for the ACOs in our sample, the results presented here show that $53 \%$ and $32 \%$ of the objects in the sample receive at least 1 collision energetic enough to break the comet crust and allow a dormant comet to reach an active state in a period shorter than a JFC dynamical lifetime, $37 \%$ and $16 \%$ receive 2 or more collisions, $26 \%$ and $11 \%$ receive at least 3 , and $16 \%$ and $7 \%$ receive not fewer than 4 , for projectile distributions with $r_{\mathrm{c}}=200 \mathrm{~m}$ and $100 \mathrm{~m}$, respectively (Fig. 1). Then, many objects in the ACOs sample received 1 or more collisions during their dynamical lifetimes and, if they are dormant comets, they could be reactivated.

On the other hand, in spite of their short dynamical lifetimes, comets become inactive mainly by physical causes due to a steady mass loss by sublimation of volatiles or by formation of a dust mantle. Based on the mean rate of secular brightness decrease, Kresák \& Kresáková (1990) have estimated a mean active lifetime of $\sim 6 \times 10^{3} \mathrm{yr}$ for JFCs with a perihelion distance less than 1.5 AU, while Fernández (1985) found a lifetime of $\sim 2 \times 10^{4} \mathrm{yr}$ for these objects. Taking $1 \times 10^{4} \mathrm{yr}$ as a working value for the mean active lifetime of JFCs before they become dormant, and assuming that each time a dormant comet receives a collision energetic enough to expose fresh ices it becomes active for at least half the initial active period $\left(5 \times 10^{3} \mathrm{yr}\right)$, it is expected to see these objects in an active state during a significant fraction of their dynamical lifetimes. As shown in Fig. 2 and Table 1, a large fraction of the largest objects in the ACO sample $\left(r_{\mathrm{t}} \geq 8-9 \mathrm{~km}\right)$ receives more than 4 collisions during this period and could be active during more than $3 \times 10^{4} \mathrm{yr}$.

Using this result we can compare the present number of objects in the active comet and ACO sample to test our initial assumption that they are dormant comets. Since the population of dormant comet candidates must be seriously affected by an observational bias, which is more serious for the smaller and fainter objects, to allow a direct comparison only the largest objects in both populations are taken into account to minimize the bias effect. Then, using the radii of JFCs obtained by Tancredi et al. (2006), we found for the active population only 1 comet with 


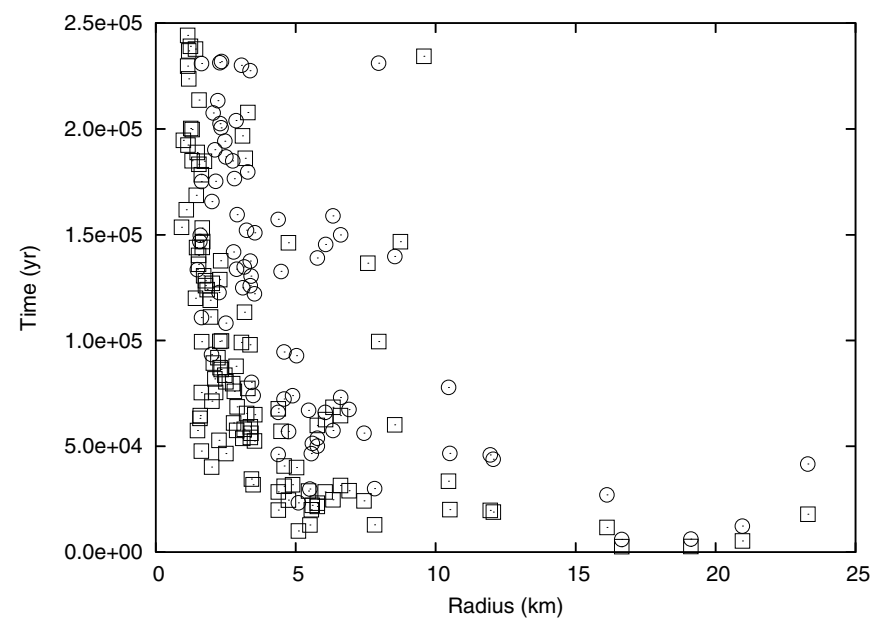

Fig. 2. Time needed to receive a collision energetic enough to reactivate a dormant comet in function of its radius. Only objects with collision time shorter than $2.5 \times 10^{5} \mathrm{yr}$ are shown. The results for the projectile distributions with $r_{\mathrm{c}}=200 \mathrm{~m}$ and $100 \mathrm{~m}$ are indicated with squares and circles, respectively.

radius larger than $9 \mathrm{~km}, 2$ objects with radius larger than $5 \mathrm{~km}$, and 5 objects with radius larger than $4 \mathrm{~km}$. Taking into account these values and the period the comets are in an active state found previously, we must expect $\sim 6, \sim 12$ and $\sim 32$ dormant comets in each radius range, respectively. In the ACO sample we found 15, 46 and 59 objects, respectively, that are in excess from the expected values and are indicative of the presence in the ACO population of objects that are not comets in a dormant state. These objects could be asteroids (possibly Hildas) with $T_{\mathrm{J}}<3$ obtaining their present orbits by some dynamical mechanism that perturbed the original asteroidal orbit changing its Tisserand invariant (Di Sisto et al. 2005).

The objects (1373) Cincinatti, (2938) Hopi, and (20898) Fountainhills receive collisions energetic enough to reactivate a dormant comet at a rate of $>1-2 \times 10^{-4} \mathrm{yr}^{-1}$, depending on the projectile size distribution. With these collision rates, they are the best candidates in the ACO sample to be observed during a reactivation if they are dormant comets.

While all the assumptions made here are reasonable, it is necessary to consider two of them more deeply. First, if the projectile size distribution used for the calculations is not accurate, the results presented here could be highly modified. Since the size distribution changes for different size ranges and for very small objects is not well known, it is not easy to choose values for their parameters in the smaller size end of the distribution. As a first guess we used the size distribution proposed by Yoshida \& Nakamura (2007) for objects with a radius between $500 \mathrm{~m}$ and 200 or $100 \mathrm{~m}$, in spite that these authors proposed it for objects larger than $250 \mathrm{~m}$. It is possible that this size distribution could be still valid for smaller objects (for example, for $r \lessgtr 100 \mathrm{~m}$ ) producing a shallow size distribution and a shortage of small projectiles, making the ACO collisional reactivation process very improbable. Nevertheless, this shallow size distribution extended to very small sizes is difficult to reconcile with the cratering records of (243) Ida, (253) Mathilde, and (951) Gaspra (Chapman 2002).

Second, the choice of the minimum exposed area on the cometary surface to be considered active during its next perihelion passage is rather arbitrary. Taking the double of the original value $(20 \%$ of the total surface area of an object with radius $1 \mathrm{~km}$ ), the radius of the projectile needed to make a crater with that dimension is 1.56 times the original radius and the time needed to receive such a collision is $2-3$ times longer, depending on the size distribution used and if the radius of the projectile is larger or smaller than $r_{\mathrm{c}}$. In this case, $37 \%$ and $16 \%$ of the objects in the sample receive at least 1 collision energetic enough to break the comet crust and allow a reactivation, and $16 \%$ and $7 \%$ receive more than 2 collisions for the projectile distributions with $r_{\mathrm{c}}=200 \mathrm{~m}$ and $100 \mathrm{~m}$, respectively. Then, the objects in the ACO sample with $r_{\mathrm{t}} \geq 8-9 \mathrm{~km}$ receive more than 2 collisions during this period and could be active during $>10 \%$ of their dynamical lifetimes, and we must expect $\sim 9, \sim 18$ and $\sim 45$ dormant comets for a comet radius of $>9 \mathrm{~km},>5 \mathrm{~km}$, and $>4 \mathrm{~km}$, respectively, which are also indicative of the presence in the ACO population of objects that are not comets in a dormant state.

\section{Conclusions}

We compute the mean intrinsic collision probability, the mean collision velocity, and the time needed to be reactivated by a collision for a sample of 206 ACOs that are dormant comet candidates from the Jupiter family.

The results presented here show that a large fraction of the objects in the sample receive at least 1 collision energetic enough to break the comet crust and allow a dormant comet to reach an active state in a period shorter than a JFC dynamical lifetime. A large fraction of the objects in the ACO sample with $r_{\mathrm{t}} \geq 8-9 \mathrm{~km}$ receive several collisions and could be active during more than $3 \times 10^{4} \mathrm{yr}$.

Three objects, (1373) Cincinatti, (2938) Hopi, and (20898) Fountainhills, receive collisions energetic enough to reactivate a dormant comet at a high rate, being the best candidates in the ACO sample of being observed during a reactivation if they are dormant comets.

Comparing only the largest objects in the active JFC population and ACO sample, we found an excess in the number of dormant comet candidates from the expected values which is indicative of the presence in the ACO population of objects that are not comets in a dormant state. These objects could be asteroids with $T_{\mathrm{J}}<3$ obtaining their present orbits by a dynamical mechanism that perturbs the original asteroidal orbit, changing its Tisserand invariant.

Acknowledgements. The authors wish to thanks J. A. Fernández for his comments and suggestions. The authors acknowledge the partial financial support by CICITCA, Universidad Nacional de San Juan, through a research grant.

\section{References}

Alvarez-Candal, A., \& Licandro, J. 2006, A\&A, 458, 1007

Beech, M. 2001, MNRAS, 327, 1201

Benkhoff, J., \& Huebner, W. F. 1996, Planet. Space Sci., 44, 1005

Bottke, W. F., Durda, D. D., Nesvorný, et al. 2005, Icarus, 179, 63

Chapman, C. R. 2002, in Asteroids III, ed. W. F. Bootke, Jr., A. Cellino, P.

Paolicchi, \& R. P. Binzel (Tucson: Univ. of Arizona Press), 315

Di Sisto, R. P., Brunini, A., Dirani, L., \& Orellana, R. B. 2005, Icarus, 174, 81

Dohnanyi, J. W. 1969, J. Geophys. Res., 74, 2531

Farinella, P., \& Davis, D. R. 1992, Icarus, 97, 111

Fernández, J. A. 1981, Moon Plan., 25, 507

Fernández, J. A. 1985, Icarus, 64,308

Fernández, J. A. 1990, in Asteroids, Comets, Meteors III, ed. C.-I. Lagerkvist, H. Rickman, B. A. Lindblad, \& M. Lindgren, Uppsala University, Uppsala, 309

Fernández, J. A., Gallardo, T., \& Brunini, A. 2002, Icarus, 159, 358

Jewitt, D. 2002, AJ, 123, 1039

Jewitt, D. 2005, AJ, 129, 530 
Kresák, L. 1974, Bull. Astron. Inst. Czechosl., 25, 293

Kresák, L. 1979, in Asteroids, ed. T. Gehrels (Tucson: Univ. of Arizona Press), 289

Kresák, L., \& Kresáková, M. 1990, Icarus, 86, 82

Kührt, E., \& Keller, H. U. 1994, Icarus, 109, 121

Licandro, J., de León, J., Pinilla, N., \& Serra-Ricart, M. 2005, Adv. Space Res. 38,1991

Marzari, F., Scholl, H., \& Farinella, P. 1996, Icarus, 119, 192

Matese, J. J., \& Whitman, P. G. 1994, Icarus, 109, 258

Morbidelli, A., \& Vokrouhlický, D. 2003, Icarus, 163, 120

Prialnik, D., \& Mekler, Y. 1991, ApJ, 366, 318

Rickman, H., Fernández, J. A., \& Gustafson, B. 1990, A\&A, 237, 524
Schenk, P. M., Chapman, C., Moore, J., \& Zahnle, K. 2004, in Jupiter: Planet, Satellites, and Magnetosphere, ed. F. Bagenal, \& W. McKinnon (Cambridge: Cambridge Univ. Press), 427

Schmidt, R. M., \& Housen, K. R. 1987, Int. J. Impact Eng., 5, 543

Tancredi, G., Fernández, J. A., Rickman, H., \& Licandro, J. 2006, Icarus, 182, 527

Tedesco, E. F., \& Desert, F. 2002, AJ, 123, 2070

Tedesco, E. F., Cellino, A., \& Zappalá, V. 2005, AJ, 129, 2869

Toth, I. 2000, A\&A, 360, 375

Toth, I. 2001, A\&A, 368, L25

Yoshida, F., \& Nakamura, T. 2007, Planet. Space Sci., 55, 1113

Zahnle, K., Dones, L., \& Levison, H. F. 1998, Icarus, 136, 202 
C. G. Díaz and R. Gil-Hutton: Collisional activation of asteroids in cometary orbits, Online Material p 1

Table 1. Physical and collisional parameters for ACOs in the dormant comet candidate sample.

\begin{tabular}{|c|c|c|c|c|c|c|c|c|c|}
\hline & $\mathrm{ACO}$ & $\mathrm{H}$ & $\begin{array}{r}r_{\mathrm{t}} \\
\mathrm{km}\end{array}$ & $\begin{array}{c}\left\langle P_{\mathrm{i}}\right\rangle \\
\mathrm{km}^{-2} \mathrm{yr}^{-1} \\
\end{array}$ & $\begin{array}{c}\left\langle V_{\text {col }}\right\rangle \\
\mathrm{km} \mathrm{s}^{-1}\end{array}$ & $\begin{array}{r}\sigma_{v_{\mathrm{col}}} \\
\mathrm{km} \mathrm{s}^{-1}\end{array}$ & $\begin{array}{l}r_{a} \\
\mathrm{~m} \\
\end{array}$ & $\begin{array}{c}T_{200} \\
\mathrm{yr} \\
\end{array}$ & $\begin{array}{c}T_{100} \\
\mathrm{yr} \\
\end{array}$ \\
\hline$(944)$ & Hidalgo & 10.77 & 23.30 & $0.16 \mathrm{E}-18$ & 17.90 & 2.80 & 172.09 & 17930 & 41636 \\
\hline (1373) & Cincinnati & 11.20 & 19.12 & $0.21 \mathrm{E}-17$ & 13.21 & 2.55 & 193.16 & 2669 & 6198 \\
\hline (1922) & Zulu & 12.20 & 12.06 & $0.52 \mathrm{E}-18$ & 13.68 & 2.89 & 166.32 & 18913 & 43919 \\
\hline (2938) & Hopi & 11.50 & 16.65 & $0.24 \mathrm{E}-17$ & 13.92 & 2.63 & 180.37 & 2595 & 6026 \\
\hline (5164) & Mullo $^{1}$ & 13.10 & 7.97 & $0.21 \mathrm{E}-18$ & 11.85 & 2.88 & 160.46 & 99502 & 231065 \\
\hline (6144) & 1994 EQ3 & 11.50 & 16.65 & $0.59 \mathrm{E}-19$ & 7.18 & 2.25 & 262.04 & 272545 & 632909 \\
\hline (7604) & Kridsadaporn & 13.70 & 6.05 & $0.99 \mathrm{E}-18$ & 12.17 & 2.78 & 146.21 & 28453 & 66075 \\
\hline$(8373)$ & Stephengould & 13.80 & 5.77 & $0.11 \mathrm{E}-17$ & 14.58 & 2.96 & 130.34 & 21606 & 50174 \\
\hline (9767) & Midsomer Norton & 16.40 & 1.74 & $0.45 \mathrm{E}-18$ & 17.74 & 4.09 & 83.24 & 184721 & 428964 \\
\hline (17493) & Wildcat & 14.30 & 4.59 & $0.99 \mathrm{E}-18$ & 14.72 & 3.26 & 121.49 & 31159 & 72357 \\
\hline (18916) & 2000 OG44 & 14.30 & 4.59 & $0.22 \mathrm{E}-18$ & 8.94 & 3.31 & 160.95 & 286006 & 664170 \\
\hline (20898) & Fountainhills & 11.00 & 20.96 & $0.73 \mathrm{E}-18$ & 15.78 & 2.66 & 179.33 & 5326 & 12367 \\
\hline (30512) & $2001 \mathrm{HO} 8$ & 12.50 & 10.51 & $0.98 \mathrm{E}-18$ & 9.42 & 2.58 & 197.44 & 20093 & 46662 \\
\hline (32511) & $2001{\mathrm{NX} 17^{3}}^{3}$ & 12.70 & 9.58 & $0.12 \mathrm{E}-18$ & 8.21 & 2.66 & 207.89 & 234349 & 544210 \\
\hline (37117) & 2000 VU2 ${ }^{4}$ & 13.20 & 7.61 & $0.18 \mathrm{E}-19$ & 7.00 & 1.90 & 213.15 & 2488569 & 5779010 \\
\hline (37384) & 2001 WU1 & 14.30 & 4.59 & $0.94 \mathrm{E}-18$ & 12.67 & 2.83 & 132.21 & 40718 & 94557 \\
\hline (41461) & $2000 \mathrm{ON}$ & 13.90 & 5.51 & $0.19 \mathrm{E}-17$ & 14.73 & 2.92 & 127.92 & 12859 & 29861 \\
\hline ( 88043) & 2000 UE110 & 14.40 & 4.38 & $0.14 \mathrm{E}-17$ & 16.11 & 3.23 & 113.97 & 19897 & 46204 \\
\hline (96177) & $1984 \mathrm{BC}^{5}$ & 16.20 & 1.91 & $0.15 \mathrm{E}-18$ & 12.38 & 3.16 & 104.66 & 827784 & 1922300 \\
\hline (101795) & 1999 HX2 & 15.30 & 2.89 & $0.13 \mathrm{E}-17$ & 12.13 & 2.75 & 119.00 & 57568 & 133687 \\
\hline (115916) & 2003 WB8 & 14.10 & 5.03 & $0.87 \mathrm{E}-18$ & 12.41 & 2.69 & 137.29 & 39967 & 92812 \\
\hline (116908) & 2004 GT2 & 14.90 & 3.48 & $0.20 \mathrm{E}-17$ & 11.47 & 2.40 & 129.36 & 31869 & 74006 \\
\hline (136620) & 1994 JC & 15.10 & 3.17 & $0.33 \mathrm{E}-18$ & 18.09 & 4.20 & 97.48 & 113411 & 263365 \\
\hline (138512) & 2000 LE3 & 13.60 & 6.33 & $0.10 \mathrm{E}-17$ & 12.72 & 2.90 & 144.48 & 24769 & 57519 \\
\hline (144870) & 2004 MA8 & 14.40 & 4.38 & $0.17 \mathrm{E}-17$ & 11.16 & 2.53 & 140.19 & 28460 & 66091 \\
\hline (145485) & 2005 UN398 & 13.80 & 5.77 & $0.65 \mathrm{E}-18$ & 10.10 & 3.36 & 160.33 & 59856 & 139000 \\
\hline \multirow[t]{19}{*}{ (145627) } & 2006 RY102 & 11.10 & 20.02 & $0.12 \mathrm{E}-20$ & 5.46 & 1.44 & 322.12 & 15975562 & 37098808 \\
\hline & $1983 \mathrm{JZ1}^{6}$ & 13.60 & 6.33 & $0.36 \mathrm{E}-18$ & 12.74 & 2.88 & 144.35 & 68440 & 158932 \\
\hline & 1989 FR & 11.57 & 16.12 & $0.79 \mathrm{E}-18$ & 10.91 & 2.63 & 205.08 & 11669 & 27099 \\
\hline & 1992 XA & 17.35 & 1.13 & $0.92 \mathrm{E}-19$ & 9.71 & 2.94 & 103.37 & 3710281 & 8616097 \\
\hline & 1995 KG1 & 19.39 & 0.44 & $0.87 \mathrm{E}-18$ & 16.22 & 4.29 & 59.38 & 644006 & 1495526 \\
\hline & 1995 WL $^{7}$ & 19.01 & 0.52 & $0.43 \mathrm{E}-18$ & 11.93 & 3.27 & 74.19 & 1612762 & 3745191 \\
\hline & 1997 GF3 & 17.43 & 1.08 & $0.74 \mathrm{E}-18$ & 14.11 & 3.13 & 82.86 & 285659 & 663363 \\
\hline & 1997 UR14 & 16.84 & 1.42 & $0.17 \mathrm{E}-17$ & 11.36 & 2.65 & 101.10 & 119978 & 278617 \\
\hline & 1998 ВС34 & 20.84 & 0.23 & $0.20 \mathrm{E}-17$ & 11.43 & 2.83 & 59.92 & 1098889 & 2551865 \\
\hline & 1998 HO121 & 12.27 & 11.68 & $0.17 \mathrm{E}-19$ & 8.07 & 1.68 & 221.97 & 1247648 & 2897316 \\
\hline & 1998 KK56 & 16.35 & 1.78 & $0.11 \mathrm{E}-17$ & 12.22 & 2.71 & 103.39 & 125975 & 292542 \\
\hline & 1998 QJ1 & 16.73 & 1.50 & $0.11 \mathrm{E}-18$ & 13.96 & 2.48 & 91.29 & 1245167 & 2891554 \\
\hline & 1998 UQ1 & 16.55 & 1.63 & $0.62 \mathrm{E}-18$ & 15.46 & 3.97 & 88.23 & 178360 & 414192 \\
\hline & 1998 WL34 & 14.16 & 4.89 & $0.65 \mathrm{E}-20$ & 5.64 & 1.04 & 212.55 & 16852414 & 39135052 \\
\hline & 1999 ТМ113 & 13.92 & 5.46 & $0.11 \mathrm{E}-17$ & 12.56 & 2.45 & 139.59 & 28856 & 67010 \\
\hline & 1999 XB69 & 16.41 & 1.74 & $0.66 \mathrm{E}-19$ & 10.84 & 2.57 & 109.77 & 2536811 & 5891039 \\
\hline & 1999 XD106 & 16.48 & 1.68 & $0.97 \mathrm{E}-18$ & 12.50 & 2.92 & 100.37 & 146770 & 340833 \\
\hline & 1999 XO188 & 13.51 & 6.60 & $0.84 \mathrm{E}-18$ & 11.76 & 3.49 & 152.79 & 31481 & 73106 \\
\hline & 2000 AC229 & 16.65 & 1.55 & $0.42 \mathrm{E}-18$ & 18.59 & 3.78 & 78.49 & 213616 & 496063 \\
\hline
\end{tabular}

\footnotetext{
${ }^{1} T_{\text {int }}=77563$ yr.

${ }^{2} T_{\text {int }}=18033 \mathrm{yr}$.

${ }^{3} T_{\mathrm{int}}=75521 \mathrm{yr}$.

${ }^{4} T_{\mathrm{int}}=54421 \mathrm{yr}$.

${ }^{5} T_{\mathrm{int}}=86800 \mathrm{yr}$.

${ }^{6} T_{\text {int }}=50501 \mathrm{yr}$.

${ }^{7} T_{\mathrm{int}}=86502 \mathrm{yr}$.
} 
Table 1. continued.

\begin{tabular}{|c|c|c|c|c|c|c|c|c|}
\hline $\mathrm{ACO}$ & $\mathrm{H}$ & $\begin{array}{c}r_{\mathrm{t}} \\
\mathrm{km}\end{array}$ & $\begin{array}{c}\left\langle P_{\mathrm{i}}\right\rangle \\
\mathrm{km}^{-2} \mathrm{yr}^{-1}\end{array}$ & $\begin{array}{c}\left\langle V_{\text {col }}\right\rangle \\
\mathrm{km} \mathrm{s}^{-1}\end{array}$ & $\begin{array}{c}\sigma_{v_{\mathrm{col}}} \\
\mathrm{km} \mathrm{s}^{-1}\end{array}$ & $\begin{array}{l}r_{a} \\
\mathrm{~m}\end{array}$ & $\begin{array}{c}T_{200} \\
\mathrm{yr}\end{array}$ & $\begin{array}{c}T_{100} \\
\mathrm{yr}\end{array}$ \\
\hline $2000 \mathrm{AU} 242^{8}$ & 13.41 & 6.91 & $0.99 \mathrm{E}-18$ & 10.66 & 3.86 & 163.61 & 29015 & 67380 \\
\hline 2000 BK2 & 16.72 & 1.50 & $0.41 \mathrm{E}-19$ & 10.03 & 2.60 & 110.16 & 5406868 & 12555950 \\
\hline 2000 CA13 & 17.90 & 0.87 & $0.49 \mathrm{E}-18$ & 11.54 & 3.09 & 87.31 & 759727 & 1764254 \\
\hline 2000 EJ37 & 13.21 & 7.58 & $0.12 \mathrm{E}-18$ & 14.69 & 3.15 & 140.13 & 136520 & 317029 \\
\hline 2000 GQ132 & 17.24 & 1.18 & $0.89 \mathrm{E}-18$ & 13.08 & 2.88 & 88.64 & 236828 & 549967 \\
\hline 2000 GH147 & 17.62 & 0.99 & $0.12 \mathrm{E}-17$ & 14.54 & 3.37 & 79.48 & 194581 & 451861 \\
\hline 2000 KD41 & 16.60 & 1.59 & $0.25 \mathrm{E}-17$ & 12.03 & 2.69 & 100.98 & 64513 & 149813 \\
\hline 2000 OZ21 & 16.33 & 1.80 & $0.83 \mathrm{E}-19$ & 11.01 & 3.55 & 109.94 & 1883303 & 4373448 \\
\hline 2000 QJ46 & 15.37 & 2.80 & $0.22 \mathrm{E}-19$ & 8.67 & 2.08 & 142.51 & 5619233 & 13049109 \\
\hline 2000 QD181 ${ }^{9}$ & 15.08 & 3.20 & $0.60 \mathrm{E}-18$ & 8.23 & 2.57 & 152.40 & 186129 & 432234 \\
\hline 2000 SB1 & 15.02 & 3.29 & $0.71 \mathrm{E}-18$ & 13.28 & 2.81 & 117.26 & 77370 & 179670 \\
\hline 2000 SL44 & 15.61 & 2.51 & $0.18 \mathrm{E}-17$ & 12.85 & 2.92 & 110.64 & 46601 & 108218 \\
\hline 2000 SO182 & 13.69 & 6.07 & $0.80 \mathrm{E}-18$ & 8.04 & 2.30 & 184.97 & 62612 & 145399 \\
\hline 2000 TG24 & 15.81 & 2.29 & $0.63 \mathrm{E}-18$ & 13.97 & 3.27 & 102.84 & 128804 & 299112 \\
\hline 2000 WT168 ${ }^{10}$ & 14.35 & 4.48 & $0.67 \mathrm{E}-18$ & 12.90 & 3.55 & 130.03 & 57129 & 132666 \\
\hline $2000 \mathrm{XO}^{11}$ & 15.54 & 2.59 & $0.18 \mathrm{E}-18$ & 10.61 & 2.92 & 124.39 & 564616 & 1311164 \\
\hline 2000 YN30 & 16.82 & 1.44 & $0.19 \mathrm{E}-18$ & 13.02 & 3.01 & 93.85 & 856170 & 1988216 \\
\hline 2000 YL90 & 13.87 & 5.59 & $0.15 \mathrm{E}-17$ & 11.73 & 2.62 & 146.02 & 22141 & 51416 \\
\hline 2001 CT20 & 15.29 & 2.91 & $0.10 \mathrm{E}-17$ & 12.47 & 2.80 & 117.31 & 68690 & 159514 \\
\hline 2001 HW18 & 17.64 & 0.98 & $0.66 \mathrm{E}-18$ & 15.16 & 4.17 & 77.43 & 329400 & 764941 \\
\hline 2001 HJ30 & 17.04 & 1.30 & $0.11 \mathrm{E}-17$ & 11.99 & 2.86 & 95.55 & 184993 & 429595 \\
\hline $2001 \mathrm{JO}$ & 14.96 & 3.38 & $0.11 \mathrm{E}-17$ & 12.38 & 2.82 & 122.95 & 54194 & 125851 \\
\hline 2001 KX67 & 16.16 & 1.95 & $0.10 \mathrm{E}-17$ & 12.44 & 2.86 & 104.92 & 119059 & 276481 \\
\hline 2001 OK17 & 18.71 & 0.60 & $0.11 \mathrm{E}-17$ & 16.06 & 3.08 & 65.23 & 345238 & 801719 \\
\hline 2001 QF6 & 14.97 & 3.37 & $0.50 \mathrm{E}-18$ & 14.05 & 3.25 & 114.33 & 97966 & 227498 \\
\hline 2001 QS145 & 16.62 & 1.58 & $0.24 \mathrm{E}-17$ & 12.82 & 2.66 & 97.17 & 63192 & 146746 \\
\hline 2001 QQ199 & 12.22 & 11.95 & $0.53 \mathrm{E}-18$ & 13.17 & 3.12 & 169.48 & 19780 & 45933 \\
\hline 2001 QG288 ${ }^{12}$ & 15.69 & 2.42 & $0.40 \mathrm{E}-18$ & 8.41 & 2.59 & 139.08 & 390587 & 907030 \\
\hline 2001 SK276 & 17.31 & 1.15 & $0.95 \mathrm{E}-18$ & 11.56 & 3.16 & 94.18 & 273585 & 635326 \\
\hline 2001 TX16 & 13.90 & 5.51 & $0.13 \mathrm{E}-18$ & 10.95 & 3.54 & 151.21 & 287255 & 667069 \\
\hline 2001 UO16 & 17.53 & 1.04 & $0.14 \mathrm{E}-17$ & 9.99 & 2.70 & 99.38 & 263461 & 611816 \\
\hline $2001 \mathrm{VE}$ & 15.05 & 3.25 & $0.12 \mathrm{E}-17$ & 10.19 & 2.69 & 135.62 & 65515 & 152140 \\
\hline 2001 WX1 & 14.95 & 3.40 & $0.11 \mathrm{E}-17$ & 11.63 & 2.82 & 127.52 & 56175 & 130452 \\
\hline 2001 XN88 & 18.62 & 0.63 & $0.12 \mathrm{E}-17$ & 14.47 & 3.29 & 69.99 & 358418 & 832327 \\
\hline 2001 XW150 & 16.78 & 1.46 & $0.48 \mathrm{E}-18$ & 9.46 & 3.48 & 112.97 & 530463 & 1231854 \\
\hline 2001 YK61 & 13.76 & 5.88 & $0.20 \mathrm{E}-20$ & 8.57 & 2.62 & 176.82 & 23498712 & 54569236 \\
\hline 2002 AA16 & 15.80 & 2.30 & $0.11 \mathrm{E}-17$ & 12.16 & 2.68 & 111.36 & 87189 & 202471 \\
\hline 2002 AW33 & 16.93 & 1.37 & $0.67 \mathrm{E}-18$ & 10.84 & 2.93 & 102.60 & 339665 & 788777 \\
\hline 2002 AO148 & 12.67 & 9.71 & $0.56 \mathrm{E}-19$ & 6.78 & 1.91 & 232.49 & 627359 & 1456867 \\
\hline 2002 JC68 & 16.50 & 1.66 & $0.91 \mathrm{E}-18$ & 12.80 & 2.97 & 98.78 & 153139 & 355623 \\
\hline 2002 JE109 & 18.57 & 0.64 & $0.19 \mathrm{E}-17$ & 9.96 & 2.88 & 86.97 & 366299 & 850627 \\
\hline 2002 JW115 & 15.81 & 2.29 & $0.59 \mathrm{E}-18$ & 17.59 & 3.54 & 90.31 & 99617 & 231334 \\
\hline 2002 KJ8 & 20.25 & 0.30 & $0.27 \mathrm{E}-17$ & 10.10 & 2.73 & 69.37 & 667800 & 1550781 \\
\hline 2002 LJ27 & 18.01 & 0.83 & $0.87 \mathrm{E}-18$ & 11.87 & 3.17 & 84.72 & 437563 & 1016120 \\
\hline 2002 MO3 & 16.55 & 1.63 & $0.17 \mathrm{E}-17$ & 18.96 & 3.01 & 78.63 & 47734 & 110849 \\
\hline 2002 OL15 & 18.27 & 0.74 & $0.18 \mathrm{E}-17$ & 8.24 & 3.12 & 100.63 & 415792 & 965562 \\
\hline 2002 PA96 & 15.14 & 3.11 & $0.13 \mathrm{E}-17$ & 12.02 & 2.82 & 122.12 & 53806 & 124950 \\
\hline 2002 QC25 & 17.14 & 1.24 & $0.81 \mathrm{E}-18$ & 13.32 & 2.91 & 88.88 & 239026 & 555071 \\
\hline
\end{tabular}

\footnotetext{
${ }^{8} T_{\text {int }}=8142$ yr.

${ }^{9} T_{\text {int }}=61536 \mathrm{yr}$.

${ }^{10} T_{\text {int }}=83083 \mathrm{yr}$.

${ }^{11} T_{\text {int }}=69831 \mathrm{yr}$.

${ }^{12} T_{\text {int }}=52223 \mathrm{yr}$.
} 
C. G. Díaz and R. Gil-Hutton: Collisional activation of asteroids in cometary orbits, Online Material p 3

Table 1. continued.

\begin{tabular}{|c|c|c|c|c|c|c|c|c|}
\hline ACO & $\overline{\mathrm{H}}$ & $\begin{array}{c}r_{\mathrm{t}} \\
\mathrm{km}\end{array}$ & $\begin{array}{c}\left\langle P_{\mathrm{i}}\right\rangle \\
\mathrm{km}^{-2} \mathrm{yr}^{-1}\end{array}$ & $\begin{array}{c}\left\langle V_{\text {col }}\right\rangle \\
\mathrm{km} \mathrm{s}^{-1}\end{array}$ & $\begin{array}{c}\sigma_{v_{\mathrm{col}}} \\
\mathrm{km} \mathrm{s}^{-1}\end{array}$ & $\begin{array}{l}r_{a} \\
\mathrm{~m}\end{array}$ & $\begin{array}{c}T_{200} \\
\mathrm{yr}\end{array}$ & $\begin{array}{c}T_{100} \\
\mathrm{yr}\end{array}$ \\
\hline 2002 RQ28 & 18.44 & 0.68 & $0.15 \mathrm{E}-18$ & 8.92 & 3.29 & 94.13 & 5080341 & 11797681 \\
\hline 2002 SU & 16.74 & 1.49 & $0.18 \mathrm{E}-17$ & 17.24 & 2.90 & 80.94 & 57479 & 133478 \\
\hline 2002 TQ65 & 15.36 & 2.81 & $0.10 \mathrm{E}-17$ & 11.89 & 2.82 & 119.41 & 76000 & 176489 \\
\hline 2002 ТT67 & 16.09 & 2.01 & $0.17 \mathrm{E}-17$ & 11.94 & 2.77 & 108.35 & 71342 & 165672 \\
\hline 2002 TV68 & 17.99 & 0.84 & $0.57 \mathrm{E}-18$ & 14.75 & 4.54 & 75.14 & 484987 & 1126248 \\
\hline 2002 TR96 & 15.99 & 2.11 & $0.16 \mathrm{E}-17$ & 10.91 & 2.39 & 115.50 & 81908 & 190208 \\
\hline 2002 TM190 & 14.16 & 4.89 & $0.12 \mathrm{E}-17$ & 11.73 & 2.80 & 140.62 & 31855 & 73974 \\
\hline 2002 UR12 & 16.10 & 2.00 & $0.17 \mathrm{E}-17$ & 17.72 & 2.96 & 86.61 & 40199 & 93352 \\
\hline 2002 UP36 & 17.13 & 1.25 & $0.26 \mathrm{E}-18$ & 7.29 & 2.33 & 125.04 & 1725249 & 4006412 \\
\hline 2002 VP94 & 17.14 & 1.24 & $0.35 \mathrm{E}-18$ & 11.99 & 3.01 & 94.32 & 634692 & 1473896 \\
\hline 2002 YK29 & 17.98 & 0.84 & $0.14 \mathrm{E}-17$ & 9.35 & 2.53 & 97.30 & 376375 & 874026 \\
\hline 2003 BL & 16.04 & 2.06 & $0.72 \mathrm{E}-18$ & 18.20 & 3.68 & 85.98 & 89383 & 207568 \\
\hline 2003 BM1 ${ }^{13}$ & 18.30 & 0.73 & $0.33 \mathrm{E}-18$ & 8.08 & 2.47 & 101.35 & 2375798 & 5517132 \\
\hline 2003 BA19 & 13.88 & 5.56 & $0.15 \mathrm{E}-17$ & 12.76 & 2.84 & 139.07 & 20064 & 46593 \\
\hline 2003 BU35 & 16.19 & 1.92 & $0.47 \mathrm{E}-18$ & 10.86 & 3.32 & 112.83 & 307978 & 715193 \\
\hline 2003 CC22 & 13.27 & 7.37 & $0.19 \mathrm{E}-19$ & 9.44 & 2.77 & 178.43 & 1644499 & 3818892 \\
\hline 2003 DA10 & 15.11 & 3.16 & $0.12 \mathrm{E}-17$ & 11.89 & 3.09 & 123.35 & 58049 & 134803 \\
\hline 2003 GB & 15.41 & 2.75 & $0.93 \mathrm{E}-18$ & 12.62 & 2.92 & 114.72 & 79628 & 184914 \\
\hline 2003 JC11 & 18.74 & 0.59 & $0.91 \mathrm{E}-18$ & 13.98 & 3.21 & 70.26 & 515614 & 1197371 \\
\hline 2003 KK20 & 17.78 & 0.92 & $0.18 \mathrm{E}-17$ & 13.46 & 2.79 & 81.31 & 153509 & 356483 \\
\hline 2003 SJ5 & 19.79 & 0.37 & $0.11 \mathrm{E}-17$ & 13.29 & 3.10 & 63.08 & 875317 & 2032680 \\
\hline 2003 SC255 & 15.61 & 2.51 & $0.12 \mathrm{E}-17$ & 11.66 & 2.79 & 116.88 & 80468 & 186863 \\
\hline 2003 TA & 18.14 & 0.78 & $0.12 \mathrm{E}-17$ & 14.83 & 3.46 & 73.47 & 258252 & 599719 \\
\hline 2003 UW & 15.89 & 2.20 & $0.31 \mathrm{E}-19$ & 7.23 & 2.37 & 147.58 & 7107220 & 16504543 \\
\hline 2003 UR267 ${ }^{14}$ & 16.53 & 1.64 & $0.13 \mathrm{E}-18$ & 7.54 & 1.95 & 132.63 & 2341997 & 5438638 \\
\hline 2003 UY283 & 15.15 & 3.10 & $0.83 \mathrm{E}-21$ & 12.37 & 0.00 & 120.00 & 78441720 & 182159104 \\
\hline 2003 VA3 & 16.44 & 1.71 & $0.12 \mathrm{E}-17$ & 11.70 & 2.71 & 104.73 & 130577 & 303228 \\
\hline 2003 XX & 16.55 & 1.63 & $0.18 \mathrm{E}-17$ & 13.23 & 2.63 & 96.33 & 75411 & 175121 \\
\hline 2003 YA & 14.94 & 3.42 & $0.20 \mathrm{E}-17$ & 11.02 & 2.57 & 131.63 & 34568 & 80275 \\
\hline 2003 YH63 & 16.36 & 1.78 & $0.28 \mathrm{E}-18$ & 13.95 & 3.98 & 95.83 & 406654 & 944341 \\
\hline 2004 AE9 & 17.39 & 1.11 & $0.31 \mathrm{E}-18$ & 11.11 & 2.67 & 95.31 & 940805 & 2184758 \\
\hline 2004 BT1 ${ }^{15}$ & 14.67 & 3.87 & $0.71 \mathrm{E}-19$ & 9.02 & 2.61 & 152.63 & 1074427 & 2495058 \\
\hline 2004 DO29 & 12.95 & 8.54 & $0.29 \mathrm{E}-18$ & 12.41 & 3.87 & 159.41 & 60167 & 139722 \\
\hline 2004 DA62 & 12.51 & 10.46 & $0.24 \mathrm{E}-18$ & 18.13 & 3.16 & 136.29 & 33506 & 77808 \\
\hline 2004 EU20 & 15.95 & 2.14 & $0.12 \mathrm{E}-17$ & 13.92 & 2.85 & 101.19 & 75468 & 175253 \\
\hline 2004 ET48 & 17.31 & 1.15 & $0.14 \mathrm{E}-17$ & 11.39 & 2.68 & 94.97 & 192328 & 446628 \\
\hline 2004 FN1 & 16.51 & 1.66 & $0.88 \mathrm{E}-18$ & 13.78 & 2.94 & 94.63 & 143986 & 334367 \\
\hline 2004 FC29 & 19.80 & 0.36 & $0.51 \mathrm{E}-18$ & 14.42 & 3.15 & 60.16 & 1640783 & 3810262 \\
\hline 2004 JD2 & 15.76 & 2.34 & $0.11 \mathrm{E}-17$ & 12.10 & 2.76 & 112.25 & 86339 & 200499 \\
\hline $2004 \mathrm{KZ7}^{16}$ & 15.32 & 2.87 & $0.72 \mathrm{E}-18$ & 13.63 & 2.80 & 111.13 & 87841 & 203986 \\
\hline 2004 LH18 & 19.54 & 0.41 & $0.95 \mathrm{E}-19$ & 8.67 & 3.04 & 82.91 & 15667835 & 36384200 \\
\hline 2004 MU7 & 14.86 & 3.54 & $0.75 \mathrm{E}-18$ & 13.44 & 4.06 & 118.91 & 65003 & 150950 \\
\hline 2004 PA44 & 13.53 & 6.54 & $0.28 \mathrm{E}-20$ & 7.41 & 0.66 & 197.75 & 18040806 & 41894764 \\
\hline 2004 RH9 & 17.04 & 1.30 & $0.84 \mathrm{E}-18$ & 14.10 & 2.99 & 87.20 & 199693 & 463732 \\
\hline 2004 RR109 & 17.42 & 1.09 & $0.78 \mathrm{E}-18$ & 20.34 & 3.14 & 67.50 & 161770 & 375667 \\
\hline 2004 RT109 & 18.45 & 0.68 & $0.26 \mathrm{E}-18$ & 11.00 & 3.84 & 83.52 & 2135891 & 4960014 \\
\hline 2004 RP111 & 17.87 & 0.89 & $0.45 \mathrm{E}-18$ & 11.82 & 3.38 & 86.48 & 792315 & 1839933 \\
\hline 2004 RW141 ${ }^{17}$ & 14.29 & 4.61 & $0.60 \mathrm{E}-19$ & 5.84 & 1.81 & 204.92 & 1878830 & 4363061 \\
\hline
\end{tabular}

\footnotetext{
${ }^{13} T_{\text {int }}=45796 \mathrm{yr}$.

${ }^{14} T_{\text {int }}=26023 \mathrm{yr}$.

${ }^{15} T_{\mathrm{int}}=79108 \mathrm{yr}$.

${ }^{16} T_{\text {int }}=57151 \mathrm{yr}$.

${ }^{17} T_{\text {int }}=55126 \mathrm{yr}$.
} 
Table 1. continued.

\begin{tabular}{|c|c|c|c|c|c|c|c|c|}
\hline $\mathrm{ACO}$ & $\overline{\mathrm{H}}$ & $\begin{array}{c}r_{\mathrm{t}} \\
\mathrm{km}\end{array}$ & $\begin{array}{c}\left\langle P_{\mathrm{i}}\right\rangle \\
\mathrm{km}^{-2} \mathrm{yr}^{-1}\end{array}$ & $\begin{array}{c}\left\langle V_{\text {col }}\right\rangle \\
\mathrm{km} \mathrm{s}^{-1}\end{array}$ & $\sigma_{v_{\mathrm{col}}}$ & $r_{a}$ & $T_{200}$ & $T_{100}$ \\
\hline 2004 RO288 & 16.45 & 1.70 & $0.82 \mathrm{E}-20$ & 7.80 & 2.62 & 131.47 & 33024586 & 76690424 \\
\hline 2004 SK & 16.79 & 1.46 & $0.11 \mathrm{E}-17$ & 13.41 & 3.24 & 92.66 & 143921 & 334216 \\
\hline 2004 TS166 & 20.45 & 0.27 & $0.27 \mathrm{E}-17$ & 9.52 & 2.50 & 69.88 & 835583 & 1940409 \\
\hline 2004 UZ & 17.25 & 1.18 & $0.12 \mathrm{E}-17$ & 10.76 & 2.59 & 98.83 & 223665 & 519401 \\
\hline 2004 XL & 17.12 & 1.25 & $0.80 \mathrm{E}-18$ & 15.09 & 3.39 & 83.06 & 200216 & 464945 \\
\hline 2004 XR17 & 16.15 & 1.96 & $0.10 \mathrm{E}-17$ & 12.63 & 3.06 & 104.16 & 111149 & 258113 \\
\hline 2004 XH50 & 16.53 & 1.64 & $0.72 \mathrm{E}-18$ & 20.72 & 2.95 & 74.99 & 99433 & 230905 \\
\hline 2004 XY100 & 15.25 & 2.96 & $0.15 \mathrm{E}-18$ & 7.80 & 2.86 & 153.65 & 888575 & 2063469 \\
\hline 2004 XA131 & 13.25 & 7.44 & $0.86 \mathrm{E}-18$ & 12.49 & 2.72 & 152.76 & 24211 & 56223 \\
\hline 2004 YW & 14.87 & 3.53 & $0.10 \mathrm{E}-17$ & 12.51 & 2.87 & 123.66 & 52564 & 122064 \\
\hline 2005 AY30 & 13.80 & 5.77 & $0.12 \mathrm{E}-17$ & 12.81 & 2.79 & 140.21 & 23152 & 53763 \\
\hline 2005 CR16 & 14.23 & 4.74 & $0.30 \mathrm{E}-18$ & 11.11 & 3.82 & 143.69 & 146213 & 339540 \\
\hline 2005 DE & 17.32 & 1.14 & $0.10 \mathrm{E}-17$ & 12.34 & 2.83 & 90.65 & 229639 & 533274 \\
\hline $2005 \mathrm{~EB} 127^{18}$ & 15.78 & 2.32 & $0.92 \mathrm{E}-18$ & 10.13 & 3.31 & 123.77 & 137724 & 319825 \\
\hline 2005 GP81 & 15.84 & 2.26 & $0.22 \mathrm{E}-17$ & 11.16 & 2.45 & 116.28 & 52839 & 122703 \\
\hline 2005 JM3 & 19.90 & 0.35 & $0.89 \mathrm{E}-18$ & 12.89 & 2.70 & 63.26 & 1174698 & 2727909 \\
\hline 2005 JC46 & 16.67 & 1.54 & $0.76 \mathrm{E}-18$ & 13.78 & 2.90 & 92.69 & 183322 & 425714 \\
\hline 2005 JE173 ${ }^{19}$ & 15.99 & 2.11 & $0.34 \mathrm{E}-18$ & 9.58 & 3.28 & 124.29 & 458994 & 1065886 \\
\hline 2005 KL8 & 16.36 & 1.78 & $0.11 \mathrm{E}-17$ & 12.02 & 2.90 & 104.23 & 128288 & 297914 \\
\hline 2005 NX43 20 & 16.44 & 1.71 & $0.92 \mathrm{E}-18$ & 8.59 & 2.78 & 124.67 & 257984 & 599096 \\
\hline 2005 NK61 & 16.47 & 1.69 & $0.60 \mathrm{E}-19$ & 8.62 & 2.99 & 123.94 & 4011633 & 9315904 \\
\hline 2005 QT176 & 17.73 & 0.94 & $0.10 \mathrm{E}-17$ & 12.48 & 2.96 & 85.41 & 291690 & 677368 \\
\hline 2005 RW9 & 16.68 & 1.53 & $0.11 \mathrm{E}-17$ & 13.53 & 2.81 & 93.53 & 136000 & 315821 \\
\hline 2005 SB216 & 12.33 & 11.36 & $0.35 \mathrm{E}-19$ & 8.85 & 2.00 & 209.08 & 560013 & 1300475 \\
\hline 2005 TC53 & 16.75 & 1.48 & $0.79 \mathrm{E}-18$ & 13.52 & 2.95 & 92.72 & 188854 & 438561 \\
\hline 2005 VX116 & 13.14 & 7.82 & $0.17 \mathrm{E}-17$ & 11.37 & 2.71 & 163.39 & 12933 & 30032 \\
\hline 2005 WY3 & 13.51 & 6.60 & $0.26 \mathrm{E}-18$ & 16.11 & 2.89 & 127.94 & 64550 & 149900 \\
\hline 2005 WS54 & 18.23 & 0.75 & $0.76 \mathrm{E}-18$ & 13.50 & 2.94 & 76.57 & 476199 & 1105840 \\
\hline 2005 XQ1 & 16.84 & 1.42 & $0.53 \mathrm{E}-18$ & 15.92 & 3.41 & 83.57 & 237755 & 552119 \\
\hline 2005 XK57 ${ }^{21}$ & 15.02 & 3.29 & $0.30 \mathrm{E}-18$ & 12.09 & 2.43 & 123.63 & 207738 & 482413 \\
\hline 2005 XV91 & 13.87 & 5.59 & $0.68 \mathrm{E}-19$ & 9.75 & 2.78 & 162.07 & 630605 & 1464404 \\
\hline 2005 YW3 & 14.96 & 3.38 & $0.99 \mathrm{E}-18$ & 12.34 & 2.75 & 123.17 & 59232 & 137551 \\
\hline 2005 YW24 22 & 15.66 & 2.45 & $0.76 \mathrm{E}-19$ & 8.97 & 2.60 & 134.64 & 1840053 & 4273012 \\
\hline 2005 YQ127 & 15.15 & 3.10 & $0.40 \mathrm{E}-18$ & 10.81 & 2.47 & 129.49 & 196735 & 456862 \\
\hline 2005 YR204 & 16.68 & 1.53 & $0.12 \mathrm{E}-17$ & 11.78 & 2.67 & 101.13 & 140237 & 325662 \\
\hline 2006 BV7 23 & 19.58 & 0.40 & $0.29 \mathrm{E}-19$ & 26.21 & 3.40 & 44.19 & 11132053 & 25851100 \\
\hline 2006 BQ55 & 16.08 & 2.02 & $0.62 \mathrm{E}-18$ & 16.00 & 3.20 & 91.98 & 126997 & 294916 \\
\hline 2006 BF208 & 14.40 & 4.38 & $0.52 \mathrm{E}-18$ & 13.95 & 2.72 & 123.61 & 67727 & 157277 \\
\hline 2006 BH257 & 13.51 & 6.60 & $0.18 \mathrm{E}-18$ & 8.14 & 3.16 & 188.03 & 252892 & 587271 \\
\hline 2006 DQ153 & 16.28 & 1.84 & $0.11 \mathrm{E}-17$ & 11.90 & 2.75 & 105.91 & 124055 & 288084 \\
\hline 2006 EA1 & 19.57 & 0.40 & $0.18 \mathrm{E}-17$ & 11.89 & 2.53 & 69.11 & 528780 & 1227944 \\
\hline 2006 ED1 & 18.58 & 0.64 & $0.71 \mathrm{E}-18$ & 15.02 & 3.06 & 68.89 & 544388 & 1264190 \\
\hline 2006 ES36 & 17.94 & 0.86 & $0.11 \mathrm{E}-17$ & 13.59 & 3.16 & 79.21 & 277825 & 645171 \\
\hline 2006 FV4 & 12.90 & 8.74 & $0.13 \mathrm{E}-18$ & 11.65 & 2.56 & 166.27 & 146705 & 340681 \\
\hline 2006 FH51 & 18.07 & 0.81 & $0.13 \mathrm{E}-17$ & 12.39 & 2.80 & 82.05 & 290534 & 674685 \\
\hline $2006 \mathrm{HS}^{24}$ & 15.18 & 3.06 & $0.40 \mathrm{E}-18$ & 17.76 & 4.04 & 97.48 & 99074 & 230071 \\
\hline
\end{tabular}

\footnotetext{
${ }^{18} T_{\text {int }}=17774 \mathrm{yr}$.

${ }^{19} T_{\mathrm{int}}=75332 \mathrm{yr}$.

${ }^{20} T_{\text {int }}=28392 \mathrm{yr}$.

${ }^{21} T_{\mathrm{int}}=49969 \mathrm{yr}$.

${ }^{22} T_{\text {int }}=50370 \mathrm{yr}$

${ }^{23} T_{\text {int }}=12766 \mathrm{yr}$.

${ }^{24} T_{\text {int }}=24073 \mathrm{yr}$.
} 
C. G. Díaz and R. Gil-Hutton: Collisional activation of asteroids in cometary orbits, Online Material p 5

Table 1. continued.

\begin{tabular}{|c|c|c|c|c|c|c|c|c|}
\hline $\mathrm{ACO}$ & $\overline{\mathrm{H}}$ & $\begin{array}{c}r_{\mathrm{t}} \\
\mathrm{km}\end{array}$ & $\begin{array}{c}\left\langle P_{\mathrm{i}}\right\rangle \\
\mathrm{km}^{-2} \mathrm{yr}^{-1}\end{array}$ & $\begin{array}{c}\left\langle\left\langle V_{\text {col }}\right\rangle\right. \\
\mathrm{km} \mathrm{s}^{-1}\end{array}$ & $\sigma_{v_{\mathrm{col}}}$ & $r_{a}$ & $T_{200}$ & $\bar{T} T_{100}$ \\
\hline 2006 HP131 25 & 18.11 & 0.79 & $0.95 \mathrm{E}-18$ & 8.06 & 2.13 & 104.03 & 739433 & 1717128 \\
\hline 2006 HT131 & 17.33 & 1.14 & $0.17 \mathrm{E}-17$ & 8.50 & 2.64 & 111.72 & 244219 & 567131 \\
\hline 2006 JO65 26 & 17.03 & 1.30 & $0.48 \mathrm{E}-18$ & 8.45 & 3.21 & 116.55 & 712295 & 1654107 \\
\hline 2006 QL39 & 13.58 & 6.39 & $0.23 \mathrm{E}-19$ & 9.99 & 1.75 & 166.00 & 1484856 & 3448167 \\
\hline 2006 RN16 & 13.90 & 5.51 & $0.45 \mathrm{E}-19$ & 7.01 & 2.55 & 194.47 & 1547351 & 3593293 \\
\hline $2006 \mathrm{SO} 134^{27}$ & 16.43 & 1.72 & $0.38 \mathrm{E}-19$ & 8.28 & 1.39 & 127.45 & 6426652 & 14924113 \\
\hline 2006 SH281 & 14.23 & 4.74 & $0.19 \mathrm{E}-17$ & 10.62 & 2.59 & 147.39 & 24573 & 57064 \\
\hline 2006 SV301 & 14.30 & 4.59 & $0.13 \mathrm{E}-18$ & 8.05 & 2.36 & 170.76 & 543258 & 1261567 \\
\hline 2006 ТP & 16.80 & 1.45 & $0.12 \mathrm{E}-17$ & 11.47 & 2.79 & 101.07 & 168601 & 391529 \\
\hline 2006 UE63 & 19.80 & 0.36 & $0.24 \mathrm{E}-18$ & 10.58 & 2.61 & 71.64 & 5539549 & 12864065 \\
\hline 2006 UJ170 & 18.14 & 0.78 & $0.11 \mathrm{E}-17$ & 13.11 & 3.11 & 78.76 & 315397 & 732421 \\
\hline 2006 WR3 & 15.64 & 2.47 & $0.66 \mathrm{E}-18$ & 17.16 & 3.45 & 93.62 & 83630 & 194208 \\
\hline 2006 WS3 & 16.97 & 1.34 & $0.22 \mathrm{E}-18$ & 13.81 & 3.52 & 89.03 & 760855 & 1766875 \\
\hline 2006 WF6 & 16.47 & 1.69 & $0.72 \mathrm{E}-18$ & 8.95 & 2.66 & 121.34 & 315861 & 733498 \\
\hline 2006 XL5 & 16.79 & 1.46 & $0.19 \mathrm{E}-18$ & 8.06 & 2.49 & 123.49 & 1691704 & 3928513 \\
\hline 2006 YC & 14.07 & 5.10 & $0.20 \mathrm{E}-17$ & 18.18 & 2.95 & 111.12 & 10024 & 23279 \\
\hline 2007 AD12 & 15.75 & 2.35 & $0.13 \mathrm{E}-17$ & 9.65 & 2.87 & 127.70 & 99807 & 231774 \\
\hline 2007 AK22 & 15.88 & 2.22 & $0.15 \mathrm{E}-17$ & 9.85 & 2.42 & 124.12 & 91869 & 213341 \\
\hline 2007 BU3 & 15.39 & 2.78 & $0.13 \mathrm{E}-17$ & 12.03 & 2.58 & 118.16 & 61073 & 141826 \\
\hline
\end{tabular}

\footnotetext{
${ }^{25} T_{\text {int }}=5480 \mathrm{yr}$

${ }^{26} T_{\text {int }}=56236 \mathrm{yr}$

${ }^{27} T_{\text {int }}=10191 \mathrm{yr}$
} 